\title{
Nervenkompressionssyndrome der Hand
}

\author{
Raphaela Verheggen
}

\author{
Medianus, Ulnaris, Radialis: 3 Nerven versorgen die Hand sowohl motorisch als auch sensi- \\ bel. Anhaltende Kompression in den anatomischen Engstellen führt zu entsprechenden \\ Ausfällen. Lesen Sie, welche diagnostischen Möglichkeiten es gibt und wie sich diese \\ Kompressionssyndrome in Symptomatik, Befund und Therapie unterscheiden.
}

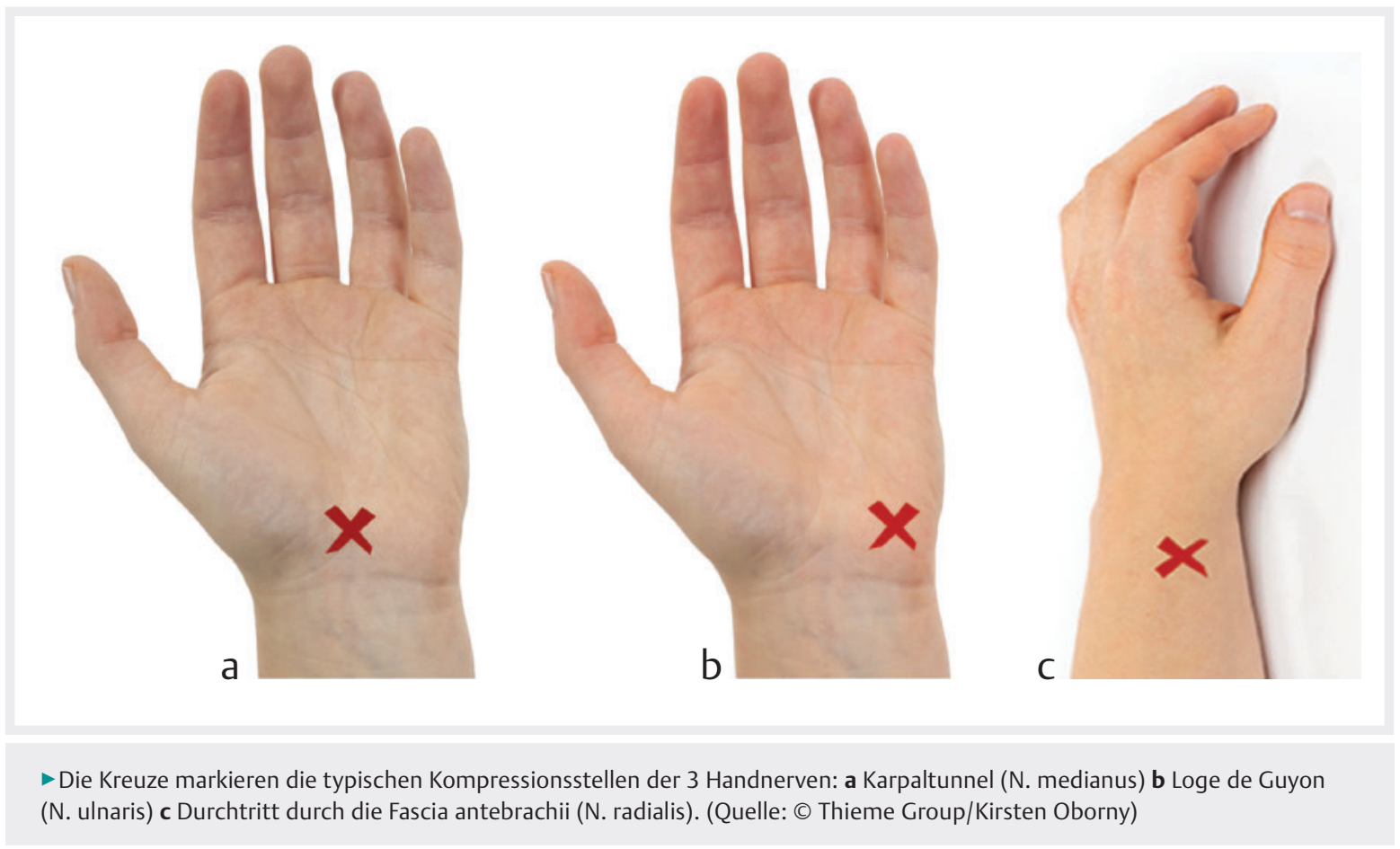

Ein Nervenkompressionssyndrom beschreibt eine funktionelle Beeinträchtigung eines oder mehrerer peripherer Nerven infolge einer chronischen Druckschädigung an anatomisch exponierten Stellen (zum Beispiel N. ulnaris am Ellbogengelenk) oder in einem osteofibrösen Kanal (zum Beispiel N. medianus bei dem Karpaltunnelsyndrom) [1] [2]. Eine Nervenkompression kann akut oder auch als chronische Schädigung (älter als 3 Monate) erscheinen. Der Schweregrad der Läsion ist abhängig vom Ausmaß und der Dauer der Kompression. Die akute Schädigung des Nervs führt zu einem Ödem und erhöhtem endoneuralen Flüssigkeitsdruck. Wird die akute Nervenkompression nicht beseitigt, entsteht infolge von strukturellen Veränderungen wie eine Fibrose eine chronische Nervenläsion [3].

Folgen dieser Druckschädigung sind Schmerzen, Funktionseinschränkungen, Sensibilitätsstörungen oder
Paresen. Ist der Nerv trotz anhaltender Kompression nicht dauerhaft geschädigt, kann ein Nervenkompressionssyndrom nach operativer Intervention teilweise oder vollständig reversibel sein.

\section{Allgemeine Diagnostik}

Im Allgemeinen ist die Annahme eines Nervenkompressionssyndroms nur dann gerechtfertigt, wenn eindeutige klinisch-neurologische oder elektrophysiologische Veränderungen des entsprechenden Nervs vorliegen [4]. Diese beiden konventionellen Methoden reflektieren den funktionellen Status des untersuchten Nervs.

\section{Schädigungshöhe und Nervenleitung}

Elektrophysiologische Untersuchungen ermöglichen eine Objektivierung der Störung, erlauben eine Lokalisation 
der Schädigung (z. B. Kompression des N. ulnaris am Ellenbogen oder in der Loge de Guyon) und gestatten eine prognostische Einordnung der Nervenschädigung, z.B. Leitungsblock (=Störung der Fortleitung des Aktionspotenzials) versus axionale Schädigung (=Schädigung der Hüllsubstanz bei einer Polyneuropathie). Mit dieser Methode lassen sich später postoperativ Regenerationsvorgänge objektivieren.

Die Elektroneurografie (ENG) ist ein gängiges Verfahren zur Untersuchung der Impulsleitung motorischer oder gemischter (enthält sensible und motorische Fasern) Nerven. Hierzu wird der zu untersuchende Nerv mittels eines kurzen elektrischen Impulses gereizt, die ausgelöste Spannungsänderung wird an einem distalen und einem proximalen Reizort gemessen. Die Differenz der Leitungszeiten (Latenz; Angabe in Millisekunden) und die Differenz der Reizorte (Angabe in Millimeter) werden ermittelt. Die Kenntnis von Abstand und Differenzzeit ermöglicht die Berechnung der Nervenleitgeschwindigkeit. Daneben werden noch Amplitude und Refraktärzeit ( = Zeitpunkt nach Auslösung eines Aktionspotenzials, in dem die Nervenzelle nicht auf einen erneuten Reiz reagieren kann) bestimmt [5].

Bei der Elektromyografie (EMG) wird elektrische Muskelaktivität (Muskelaktionspotenziale, MAP) ausgewählter Muskeln in Ruhe und bei Muskelanspannung untersucht. Hierzu werden dünne Nadelelektroden durch die Haut in einen ausgewählten Muskel eingeführt und die elektrische Aktivität wird gemessen. Bei spezifischen Fragestellungen können auch somatosensibel evozierte Potenziale (SEP) oder die motorisch evozierten Potenziale nach Magnet- oder Hochvoltstimulation (MEP) abgeleitet werden. Dabei wird nach Stimulation von sensiblen oder motorischen Fasern die Reizweiterleitung im Gehirn gemessen [5].

\section{Morphologischer Zustand des Nervs}

Die Anamneseerhebung, die neurologische und die elektrophysiologische Untersuchung sind Methoden der konventionellen Diagnostik. Zur Abklärung des morphologischen Zustands eines Nervs benötigt man entweder die Magnetresonanztomografie (MRT) oder die Neurosonografie. Mit der Einführung hochfrequenter Schallsonden $(10-14 \mathrm{MHz})$ konnten hochauflösende sonografische Darstellungen peripherer Nerven realisiert werden [6]. Mittlerweile ist die Aussagekraft der Neurosonografie verglichen mit der MRT-Bildgebung gleichwertig [7].

Ein besonderer Vorteil der Neurosonografie ist die dynamische Untersuchung unter Bewegung der betroffenen Gliedmaße und der Einsatz der Farbduplexsonografie zur Beurteilung der vaskulären Versorgung eines Nervs. Der gesunde periphere Nerv ist in den transversalen Schnitten rund bis oval und weist eine wabenförmige Echotextur auf. Auf den Longitudinalschnitten zeigt sich eine typische faszikuläre Echotextur [6]. Die wichtigsten Befunde, insbesondere bei Engpasssyndromen, sind die lokale Verdickung (typischerweise proximal, manchmal auch distal der Kompression) und z.B. die Abplattung des Nervs im Bereich des Retinaculum flexorum beim Karpaltunnelsyndrom [7]. Allgemein akzeptiert ist die Bestimmung der Querschnittsfläche (Cross Sectional Area, CSA) der faszikulären Strukturen eines Nervs unter Aussparung des echoreichen Perineuriums zur Beschreibung der Verdickung eins Nervs [8][9]. Zudem konnte gezeigt werden, dass eine lokal vergrößerte CSA mit dem Ausmaß der axonalen Schädigung des Nerves korreliert [7].

Die Magnetresonanztomografie (MRT) kann bei der Abklärung unklarer peripherer Nervenläsionen eingesetzt werden: zur Analyse des Läsionsmusters peripherer Nerven (z.B. uni-/multifokal), bei partiellen faszikulären Nervenläsionen oder zur Visualisierung von Ursachen der Nervenläsion [10]. Letzteres umfasst beispielsweise Muskelanomalien und infektiöse oder raumfordernde Prozesse. Als MRT-Kriterien für ein Karpaltunnelsyndrom gelten: Verbreiterung des N. medianus proximal des Karpaltunnels, Abflachung des N. medianus im Karpaltunnel, ein perineurales Ödem und palmare Spannung des Retinaculum flexorum [11]. Ein hochauflösendes MRT ist für den Fall eines postoperativ unbefriedigenden Behandlungsverlaufs oder bei Rezidiven sinnvoll.

\section{Karpaltunnelsyndrom (N. medianus)}

Das Karpaltunnelsyndrom (KTS) ist das mit Abstand häufigste und wichtigste Nervenkompressionssyndrom [2]. Es ist charakterisiert als eine Einengung des N. medianus im Bereich der Handwurzel. Der Karpaltunnel kann als bindegewebig umfasste Röhre am Übergang vom Unterarm zur Hand auf der palmaren Seite des Handgelenkes beschrieben werden [12]. Boden und Seitenwände des Tunnels werden durch Handwurzelknochen gebildet, und das Dach durch ein breites Band, das Retinaculum flexorum, das sich zwischen den Handwurzelknochen ausspannt. Mit dem $\mathrm{N}$. medianus verlaufen insgesamt 9 Beugesehnen (je 4 der oberflächlichen und tiefen Fingerbeuger) sowie eine Sehne des langen Daumenbeugers durch den Karpaltunnel ( $>$ Abb. 1). Das Karpaltunnelsyndrom tritt in der Regel beidseits auf, wobei die dominante Hand häufiger und stärker betroffen ist [1].

Epidemiologie Die Inzidenz der Neuerkrankungen beim KTS wird mit 3,3-3,45 auf 1000/Jahr angegeben [2][13] [14]. Männer sind deutlich seltener betroffen als Frauen (Verhältnis von 1:3,65-4) [4][15]. Der Erkrankungsgipfel liegt zwischen dem 40. und 70. Lebensjahr. Übergewichtige erkranken häufiger als Normgewichtige [4][15]. 


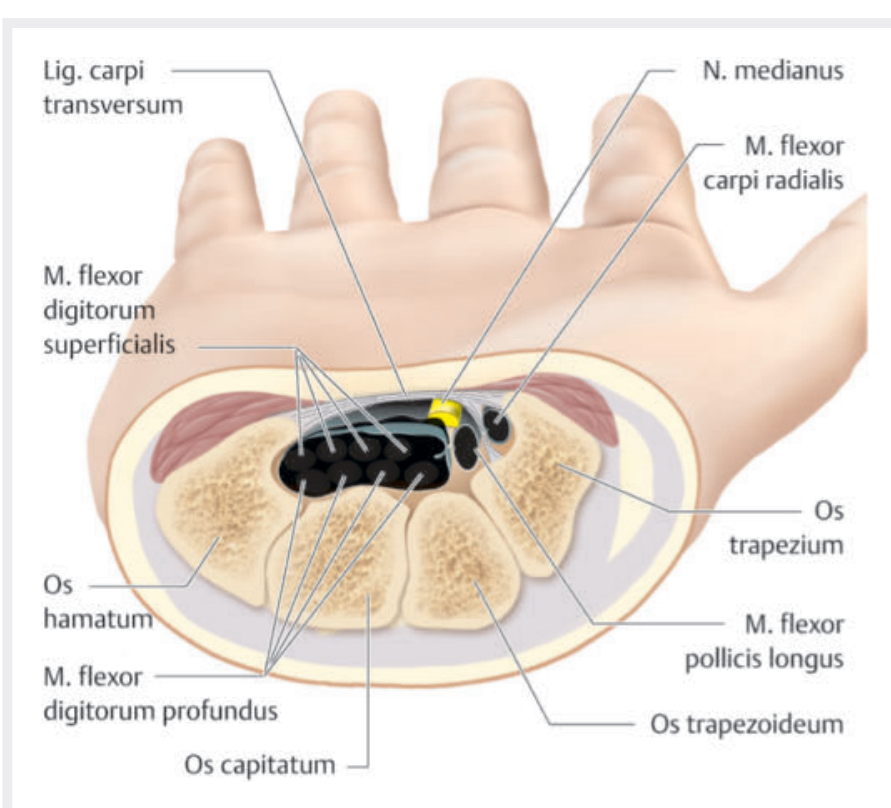

- Abb. 1 Der N. medianus läuft gemeinsam mit den Beugesehnen der Finger und des Daumens durch den Karpaltunnel, der von den Handwurzelknochen und dem Retinaculum flexorum gebildet wird. (Quelle: (c) Brucha T. Karpaltunnel, Loge de Guyon und die Sehnenfächer der Extensoren. In: Amberger R, Hrsg. Integrative Manuelle Therapie. 1. Auflage. Stuttgart: Thieme; 2017)

Ätiologie Ursache der distalen Medianuskompression ist die Volumenzunahme im Karpaltunnel oder eine traumatische Schädigung der Handwurzelknochen. Die Volumenzunahme kann unter anderem Folge einer Beugesehnen-Synovialitis, einer Tendovaginitis stenosans, eines Ödems bei Stoffwechselstörungen und Endokrinopathien (Diabetes, Akromegalie, Amyloidose) oder Blutungen infolge von Gerinnungsstörungen sein [11]. In $5-10 \%$ der Fälle tritt ein KTS im 3. Trimenon der Schwangerschaft auf. Als seltene Ursachen gelten akzessorische Muskeln oder ein atypischer Verlauf des M. palmaris longus. Gehäuft ist ein Karpaltunnelsyndrom außerdem bei Patienten mit einer chronischen Polyarthritis oder chronischer Dialysebehandlung (20\% nach einer Dialysezeit $>6,8$ Jahre) [11]. Berücksichtigt werden müssen auch mechanische Faktoren am Arbeitsplatz, z. B. bei Mitarbeitern in Gesundheitsberufen, Sekretärinnen oder infolge von Montagearbeiten oder Arbeiten an vibrierenden Geräten [5].

Symptome/Diagnostik Früh- und Kardinalsymptom beim KTS ist das schmerzhafte Einschlafen der Hände vor allem in der Nacht (Brachialgia paraesthetica nocturna). Diese Parästhesien verbessern sich durch Ausschütteln der Hände. Überdies beklagen die Patienten eine morgendliche Häufigkeit und/oder Schwellung der Finger. Pathogenetisch wird ein intraneurales Ödem mit fokaler Demyelinisierung angenommen [4]. Diskutiert wird ferner ein unwillkürliches Abknicken der Hand im Schlaf und damit Druckerhöhung im Karpaltunnel. Im weiteren Krankheitsverlauf geben Betroffene ein anhaltendes Taubheitsgefühl der Finger I-III (IV) sowie eine Störung der Feinmotorik und Geschicklichkeit an. Erst im Endstadium zeigt sich eine Atrophie der Thenarmuskulatur.

Die Verdachtsdiagnose KTS lässt sich durch Provokationsmethoden erhärten. Dazu zählt das Hoffmann-TinelZeichen mit Beklopfen der volaren Seite des Handgelenks. Bei Druckschädigung des N. medianus im Karpaltunnel verspürt der Untersuchte unangenehme, elektrisierende Parästhesien. Beim Phalen-Test wird das Handgelenk des Patienten passiv stark nach volar (oder nach dorsal) gebeugt gehalten [16]. Nach 30-40 Sekunden treten Missempfindungen im Versorgungsgebiet des N. medianus auf.

Bei typischer Anamnese und klinischem Befund ist eine elektroneurografische Untersuchung zur endgültigen Diagnosesicherung erforderlich. Bildgebende Verfahren wie die Neurosonografie und die Kernspintomografie können diese ergänzen.

Konservative Therapie Im Stadium der Brachialgie paraesthetica nocturna sollte zunächst ein konservativer Behandlungsversuch erfolgen. Befriedigende Ergebnisse werden durch Physiotherapie oder durch nächtliches Anlegen einer volaren, gepolsterten Schiene erzielt [17]. Dem Geübten gelingt es, mit Injektion von Hydrocortison im Karpalkanal eine deutliche Besserung der Beschwerden zu erzielen [18][19]. Eine einmalige lokale Steroidinfiltration hat eine begrenzte, etwa 3 Monate anhaltende Wirkdauer. Die orale Gabe von Steroiden bei leichtem Karpaltunnelsyndrom wurde ebenso in Doppelblindstudien belegt [20].

Operative Therapie Die Indikation zur operativen Intervention ist bei anhaltenden sensiblen und/oder motorischen Defiziten, Beeinträchtigungen der Stereoästhesie, Nachlassen der Abduktion und Oppositionsfähigkeit des Daumens und bei ausbleibendem Erfolg konservativer Maßnahmen gegeben. Mehrere randomisierte kontrollierte Studien belegen die Überlegenheit der operativen gegenüber der konservativen Behandlung, wobei dies unabhängig ist von der gewählten Operationsmethode: offene Spaltung versus endoskopisches Verfahren [1] [21][22]. Zu den Risiken der Operation gehören die Verletzung des Thenarastes (Ast des $\mathrm{N}$. medianus) und des Arcus palmaris superficialis (Gefäßbogen zwischen A. ulnaris und A. radialis), die inkomplette Spaltung des Retinaculum flexorum sowie das komplexe regionale Schmerzsyndrom (CRPS).

Das Behandlungsziel besteht immer in einer vollständigen Spaltung des Retinaculum flexorum (auch proximal der Rascetta), einschließlich des Lig. carpi palmare [2]. Die Therapie der Wahl ist die offene Spaltung des 
Retinaculum flexorum: Sie ist die einfachste und am häufigsten angewandte Methode. Im Hinblick auf die Spätergebnisse ist das offene Verfahren gegenüber der Endoskopie ebenbürtig [2][22]. Bei der endoskopischen Methode ist die Komplikationsrate von der Erfahrung des Operateurs abhängig. Bei inadäquater Technik und schlechter intraoperativer Übersicht besteht das Risiko der inkompletten Spaltung des Retinaculums und damit auch einer erhöhten Rezidivrate [23]. Die i.d.R. ambulant durchgeführte Operation ist auch in der Schwangerschaft, bei Diabetikern, Dialysepatienten und sehr alten Patienten angezeigt [1].

Die Nachbehandlung besteht aus einer frühfunktionellen Behandlung und Hochlagerung des Armes und der Hand zur Vermeidung einer postoperativen Schwellung. Physiotherapeutische Maßnahmen können dies ergänzen.

\section{Loge-de-Guyon-Syndrom (N. ulnaris)}

Das Loge-de-Guyon-Syndrom ist eine Druckschädigung des N. ulnaris auf Höhe des Lig. pisohamatum. Benannt wurde dieses Kompressionssyndrom nach dem Erstbeschreiber Jean Casimir Felix Guyon (1831-1920), einem französischen Urologen. Die Loge de Guyon ist eine physiologische Enge der Handwurzel zwischen dem Os pisiforme (Erbsenbein) und dem Hamulus ossis hamati (Hakenfortsatz des Hakenbeins), durch die die Äste des N. ulnaris und der begleitenden A. ulnaris verlaufen ( $\triangleright$ Abb. 2).

Ätiologie Die häufigsten Ursachen des Loge-de-GuyonSyndroms sind direkte Traumen wie Schnitt-, Schlag-, Stichverletzungen, Ganglien und intraneurale Zysten. Berufs- oder beschäftigungsbedingte Kompression oder externe Druckläsionen (Radfahrer-Lähmung, Krückenverletzung) können ebenso zu diesem Krankheitsbild führen. Beschrieben sind ferner Thrombosen der Arteria/ Vena ulnaris.

Symptome/Diagnostik Die Symptomatik bei diesem Kompressionssyndrom ist abhängig vom Läsionsort. Gross und Gelbermann (1985) [24] unterscheiden 3 Arten.

- Typ I Schädigung des N. ulnaris proximal der Loge de Guyon mit Schädigung der sensiblen (R. superficialis) und motorischen (R. profundus) Fasern des N. ulnaris. Die Läsion führt zu einer Atrophie des M. interosseus dorsalis I, Krallenstellung durch Parese der Mm. interossei und der Mm. lumbricales III / IV sowie zu Gefühlsstörungen, die die Streckseite die Finger IV/V betreffen und volarseits Finger IV (Außenseite) und V. Das Froment-Zeichen ist positiv (Papierstreifentest mit Beugung im Daumenendgelenk, siehe Info-Box).

- Typ II Isolierte Schädigung des Ramus profundus distal der Nervenaufzweigung führt zu rein motorischen Ausfällen, während sensible Störungen fehlen.
- Typ III Die Schädigung des Ramus superficialis hat rein sensible Defizite zur Folge.

Durch die elektrophysiologische Untersuchung lässt sich eine genaue Differenzierung des Lähmungstyps vornehmen. Die hochauflösende Neurosonografie ermöglicht eine Darstellung von Ganglien oder auch von Fremdkörpern (z. B. Glassplittern) als echoarme, den Nerven komprimierende Strukturen [6].

\section{FROMENT-ZEICHEN}

Der Patient hält einen Papierstreifen zwischen Daumen und Zeigefinger fest, während der Therapeut versucht, das Papier herauszuziehen. Beugt der Patient beim Festhalten das Daumenendgelenk, ist das Zeichen positiv: Der medianusinnervierte M. flexor pollicis longus kompensiert den Ausfall des ulnarisinnervierten $\mathrm{M}$. adductor pollicis. Der motorische R. profundus des N. ulnaris ist geschädigt.

Konservative Therapie Eine konservative Therapie bietet sich bei intermittierenden Beschwerden an: Beseitigung des schädigenden Reizes, Tragen einer gepolsterten Schiene nachts und Einnahme von nichtsteroidalen Antirheumatika (NSAR).

Operative Therapie In Vollnarkose, Lokalanästhesie oder Armplexusanästhesie erfolgt ulnarseitig eine bajonettförmige Hautinzision radial der Sehne des M. flexor carpi ulnaris, die sich über die Rascetta nach distal erstreckt ( $\triangleright$ Abb.3a). Nach Durchtrennung der Unterarmfaszie

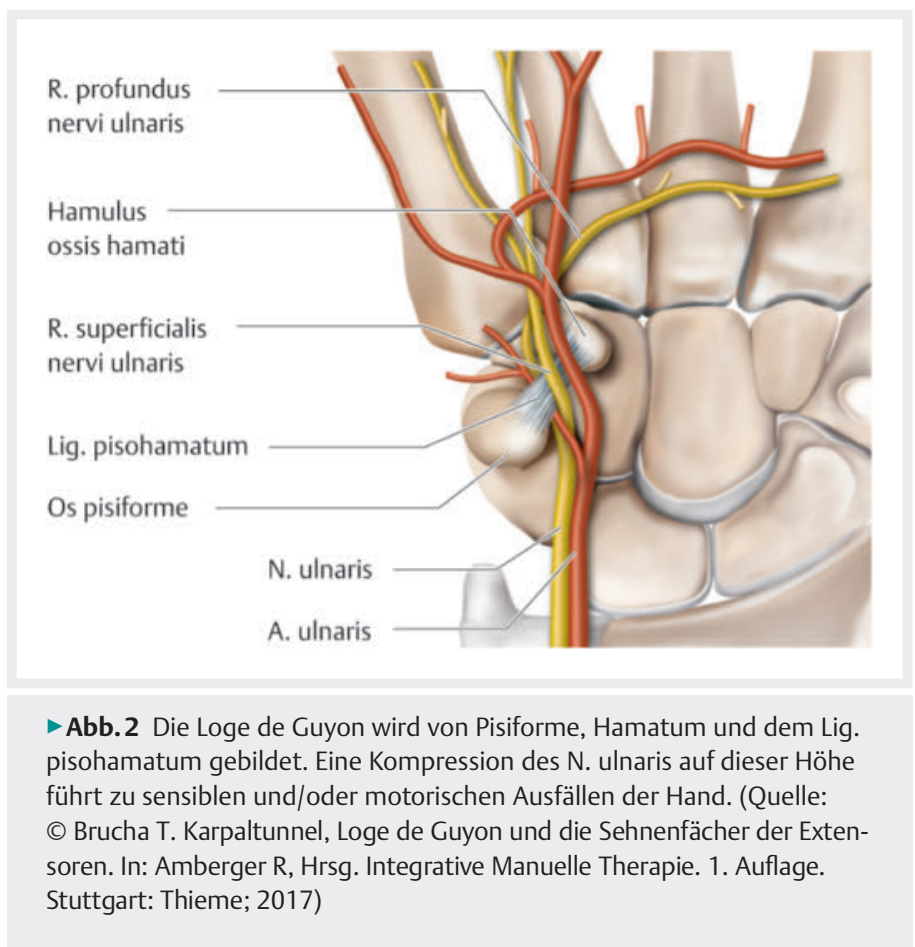



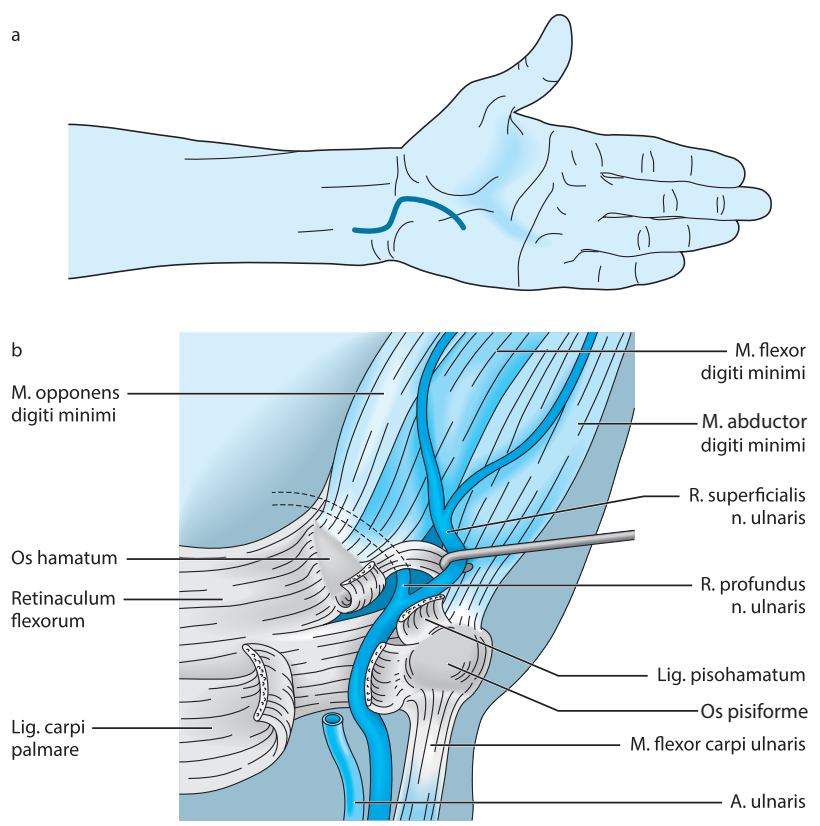

Abb. 3 Operative Eröffnung der Loge de Guyon: a Schnittführung; b Freilegung des $\mathrm{N}$. ulnaris nach Spaltung des Logendaches. (Quelle: (c) Eisenschenk A, Lautenbach M. Operative Therapie. In: Martini A, Hrsg. Orthopädie und Orthopädische Chirurgie - Ellenbogen, Unterarm, Hand. 1. Auflage. Stuttgart: Thieme; 2003)

werden der N. ulnaris und die A. ulnaris aufgesucht und nach distal verfolgt. Anschließend durchtrennt der Chirurg das Lig. carpi palmare und stellt die Aufteilungsstelle des N. ulnaris dar ( $>$ Abb.3b). Es folgt die Inspektion und Dekompression des R. profundus und Einkerben des
M. opponens digiti minimi. Die postoperative Prognose bezüglich Rückbildung motorischer Ausfälle ist bei zeitnaher Intervention gut [4]. Physiotherapeutische Maßnahmen unterstützen den Heilungsverlauf.

\section{Wartenberg-Syndrom (N. radialis)}

Die Cheiralgia paraesthetica oder Wartenberg-Syndrom benannt nach dem deutschen Neurologen und Erstbeschreiber Robert Wartenberg (1886-1956) - bezeichnet eine Kompression des Ramus superficialis $\mathrm{n}$. radialis.

Ätiologie Die Läsion betrifft ausschließlich den oberflächlichen Ast des N. radialis. Der Ort der Druckschädigung ist der distale Unterarm [25]. Der R. superficialis tritt zwischen dem M. brachioradialis (BR) und M. extensor carpi radialis longus (ECRL) durch die Fascia antebrachii ( Abb.4). In Höhe des 1. Strecksehnenfaches können die Äste des N. radialis durch externen Druck z. B. durch enge Uhrbänder, Gipsverbände oder Handschellen, aber auch nach Schlag- und Stichverletzung (iatrogen nach Venenpunktion) lädiert werden [4]. Nach Shunt-Operation zwischen $A$. radialis und $V$. cephalica antebrachii bei Dialysepatienten können sensible Ausfälle auftreten.

Symptome/Diagnostik Das Kompressionssyndrom ist gekennzeichnet durch Schmerzen und Parästhesien im Ausbreitungsgebiet des $\mathrm{R}$. superficialis $\mathrm{n}$. radialis, also am radialen Handrücken, des ersten Interdigitalraumes und am Daumen. Bei diesem Kompressionssyndrom wurden über umschriebene Druckdolenzen berichtet. Differenzialdiagnostisch muss an eine Tendovaginosis stenosans de Quervain gedacht werden,

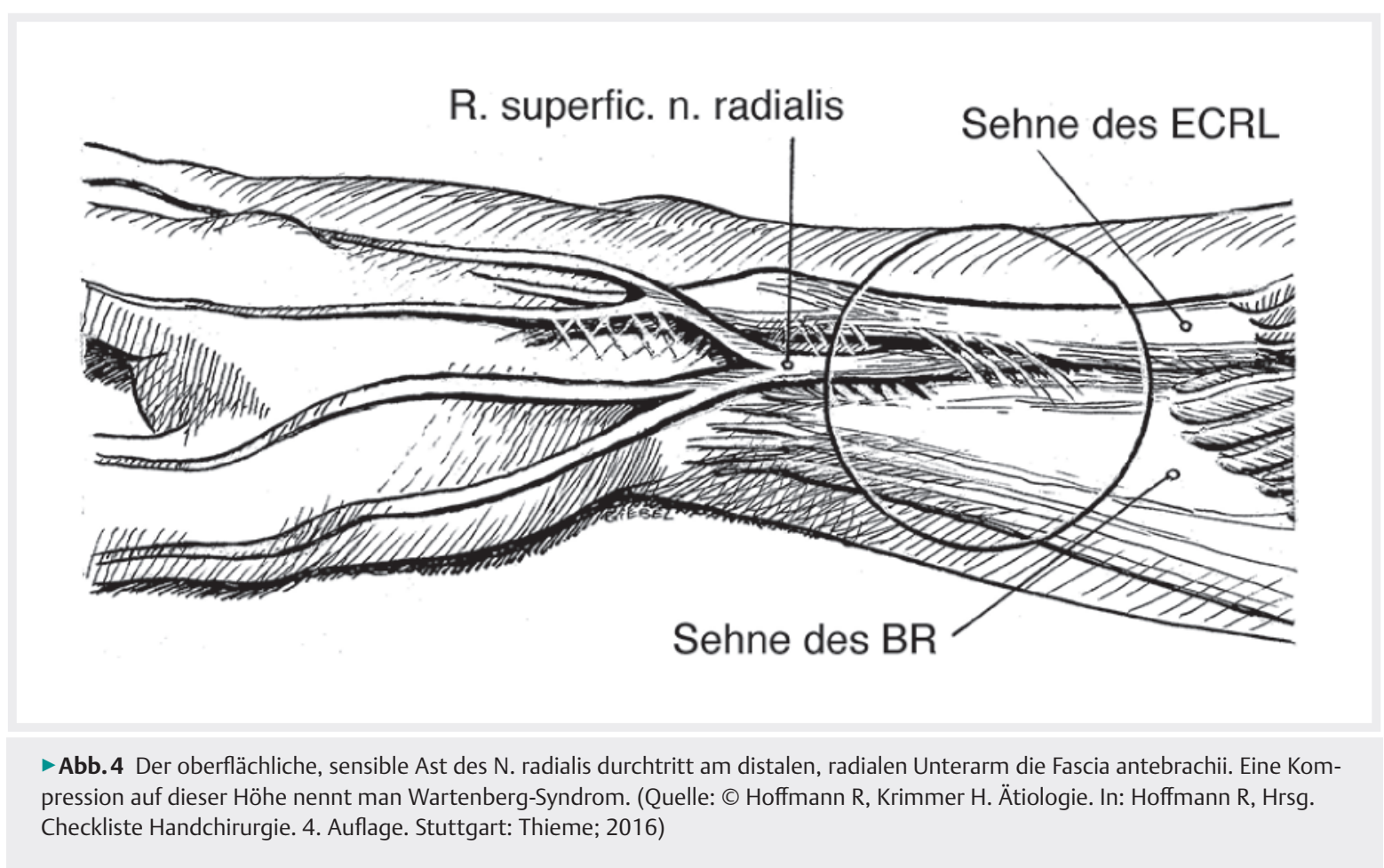


eine chronische Entzündung des 1. dorsalen Sehnenfaches der Hand.

Der klinische Verdacht einer Kompression des R. superficialis n. radialis kann durch eine diagnostische Blockade mit einem Lokalanästhetikum erhärtet werden. Die elektroneurografische Untersuchung bietet oftmals nur eine eingeschränkte diagnostische Hilfestellung. Eine neurosonografische Untersuchung kann bei diesem Krankheitsbild sinnvoll sein.

Therapie Die konservative Therapie besteht in der Elimination von äußeren Ursachen, die den R. superficialis traumatisieren. Ferner besteht die Möglichkeit einer Infiltration mit Kortikoiden oder Gabe von Antiphlogistika.

Bleibt unter konservativer Behandlung eine Besserung der Beschwerden aus, kann in Lokalanästhesie über eine radiopalmare Längsinzision unter Schonung der Hautäste des R. cutaneus antebrachii dorsalis eine Faszienspaltung vorgenommen werden mit anschließender Neurolyse des R. superficialis [4].

\section{Autorinnen/Autoren}

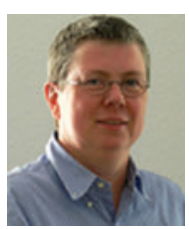

\section{Prof. Dr. Raphaela Verheggen}

Fachärztin der Neurochirurgie, Professorin und Chefärztin der neurochirurgischen Abteilung des Ev. Bathildiskrankenhaus Bad Pyrmont. Sie hat außerdem den Abschluss Master of Business Administration (MBA) - Health Care Management.

\section{Korrespondenzadresse}

AGAPLESION EV. BATHILDISKRANKENHAUS
Klinik für Neurochirurgie
Maulbeerallee 4
31812 Bad Pyrmont
Deutschland

\section{Literatur}

[1] Assmus H, Antoniadis G, Hrsg. Nervenkompressionssyndrome. Heidelberg: Springer; 2015

[2] Assmus H, Antoniadis G, Bischoff C. Karpaltunnel-, Kubitaltunnel- und seltene Nervenkompressionssyndrome. Dtsch Arztebl Int 2015; 112: 14-26

[3] Lundborg G. Nerve injury and repair. 2nd Edition. Churchill Livingstone: Elsevier; 2005

[4] Assmus H. Nervenkompressionssyndrome. Diagnostik und Chirurgie. Heidelberg: Springer; 2003

[5] Mumenthaler M, Stöhr M, Müller-Vahl H, Hrsg. Läsionen peripherer Nerven und radikuläre Syndrome. Stuttgart: Thieme; 2003
[6] Reimers CD, Gaulrapp H, Kele H. Sonographie der Muskeln, Sehnen und Nerven: Untersuchungstechnik und Befundinterpretation. Köln: Deutscher Ärzteverlag; 2004

[7] Bäumer T, Grimm A, Schelle T. Diagnostische Nervensonographie. Radiologie 2017; 57: 157-165

[8] Cartwright MS, Passmore LV, Yoon JS et al. Cross-sectional area reference values for nerve ultrasonography. Muscle Nerve 2008; 37: 566-571

[9] Roll SC, Case-Smith J, Evans K. Diagnostic accurancy of ultrasonography vs. electromyography in carpal tunnel syndrome: A systematic review of literature. Ultrasound Med Biol 2011; 37: $1539-1553$

[10] Godel T, Weiler M. Klinische Indikationen hochauflösender MRT-Diagnostik des peripheren Nervensystems. Radiologe 2017; 57: 148-156

[11] Mamoli B, Richter HP in Grisold W, Berlit P, Hrsg. Aktuelle Therapie in der Neurologie. XXVII Nervenkompressionssyndrome. 10. Erg. Lfg. Ecomed Medizin; 2007

[12] Wikipedia. Karpaltunnelsyndrom. Im Internet: https://de.wiki pedia.org/wiki/Karpaltunnelsyndrom (Zugriff am 23.1.2021)

[13] Nordstrom DL, DeStefano F, Layde PM. Incidence of diagnosed carpal tunnel syndrome in a general population. Epidemiology 1998; 9: 342-345

[14] Mondelli M, Giannini F, Giacchi M. Carpal tunnel syndrome incidence in a general population. Neurology 2002; 58: 289-94

[15] Geoghegan JM, Clark DI, Bainbridge LC et al. Risk factors in carpal tunnel syndrome. J Hand Surg Br 2004; 29: 315-320

[16] Phalen GS. The carpal-tunel syndrome. Seventeen years" experience in diagnosis and treatment of six hundred fiftyfour hands. J Bone Joint Surg (Am) 1966; 48: 211-228

[17] O'Connor D, Marshall S, Massey-Westropp N. Non surgical treatment (other than steroid injection) for carpal tunnel syndrome. Cochrane Database Syst Rev 2003; 1: CD003219. doi: 10.1002/14651858.CD003219.

[18] Dammers JWHH, Veering RMM, Vermeulen M. Injection with methylprednisolone in patients with carpal tunnel syndrome: A randomised double blind trial testing three different doses. J Neurol 2006; 253: 574-577

[19] Green DP. Diagnostik and therapeutic value of carpal tunnel injection. J Hand Surg 1984; 9A: 850-854

[20] Chang MH, Chiang HT, Lee SS et al. Oral drug of choice in carpal tunnel syndrome. Neurology 1998; 51: 390-393

[21] Verdugo RJ, Salinas RS, Castillo J, Cea JG. Surgical versus nonsurgical treatment for carpal tunnel syndrome. Cochrane Database Syst Rev 2003; 3: CD001552. doi: 10.1002/ 14651858.CD001552

[22] Vasiliadis HS, Georgoulas P, Shrier I et al. Endoscopic release for carpal tunnel syndrome. Cochrane Database Syst Rev 2014; 3: CD008265. doi: 10.1002/14651858.CD008265.pub2

[23] MacDermid JC, Richards RS, Roth JH. Endoscopic versus open carpal tunnel release: A randomized trial. J Hand Surg 2003; 28: $475-480$

[24] Gross MS, Gelberman RH. The anatomy of the distal ulnar tunnel. Clin Orthop Relat Res 1986; 196: 238-247

[25] Wartenberg R. Cheiralgia paraesthetica (Isolierte Neuritis des Ramus superficialis nervi radialis). Z f d g Neur Psych 1932; 141(1): 145-155 